\title{
Application of Multi-agents in Control of Hydrogen Powered Car to Optimize Fuel Consumption
}

\author{
Bohumil Horak, Jiri Koziorek, and Vilem Srovnal \\ VSB - Technical University of Ostrava, FEECS, Department of Measurement and Control, \\ 17. listopadu 15, 70833 Ostrava-Poruba, Czech Republic \\ \{bohumil.horak, jiri.koziorek, vilem.srovnal\}@vsb.cz
}

\begin{abstract}
Mobile embedded systems belong among typical applications of the distributed systems control in real time. An example of a mobile control system is the hydrogen powered prototype car control system. The design and realization of such distributed control system represent demanding and complex task of real time control for minimization of race car fuel consumption. The design and realization of distributed control system, mention above, is prepared for testing as a complex laboratory task. The control system software uses multiagent technology with dynamic mutual negotiation of mobile system parts. The real hardware and software model is also important motivation for extended study.
\end{abstract}

Keywords: Vehicle Control System, Distributed Control System, Multi-agents, Learning.

\section{Introduction}

A team of several specialists and students of Department of Measurement and Control, VSB-Technical University of Ostrava has designed and realized a prototype of hydrogen powered car based on fuel cell technology and electrical DC drive. The project is called HydrogenIX (Figure 1 shows a car) and the works and testing activities came through between October 2004 and today.

The motivations for the project are following:

- The development of mentioned race car is the first application of mobile system with the fuel cell in "Laboratory of Fuel Cells".

- Activate the interest of students, Ph.D. students, specialists and public in renewable and alternative energy sources.

- Enlarge cooperation between university and external subjects in the field of renewable and alternative energy sources and related technologies.

- Demonstrate results of the project in energy consumption economization at mobile vehicles.

The Shell Eco-Marathon competition is organized by Shell Company and takes place at the race circuit in Nogaro, France. Teams from all over the Europe compete to have lowest consumption of the fuel. Even if the majority of teams use petrol engines in their vehicles, there are also vehicles powered by diesel, LPG, CNG, hydrogen and 


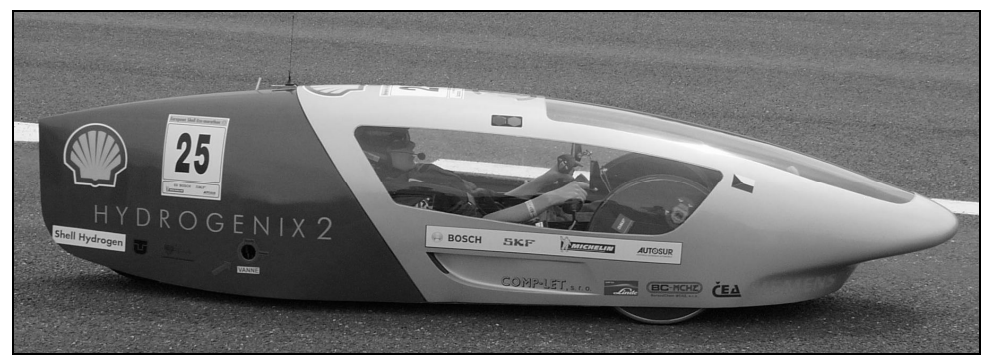

Fig. 1. The HydrogenIX car

other alternative energies. The results are obtained by the calorific value recalculating for each types of fuel. Therefore, it is possible to compare different types of fuel.

\section{Control System}

The vehicle powered by electricity generator with hydrogen fuel cell needs the electronic control system that provides control for all its parts. The complex control is necessary for basic vehicle operations and for many subsystems that have to be coordinated and controlled [6]. The control system realizes especially following tasks:

- Control of fuel cell operations - hydrogen input valve control, combustion products output valve control, fuel cell fan control, coupling of produced electrical energy to electric DC-drive system.

- DC drive control - motor current control, speed control.

- Safety and security of the car - safety of the fuel cell system and drive system, processing of hydrogen detector information, temperature measuring, etc.

- Driver control panel - complete interface to pilot that allows controlling the car start/stop, speed set point, time measuring, emergency buttons and indicators.

- Data archives with saved process variables - saving important process data to archives for next export and analyze.

- Display actual data in car - display panel in the car is a "process" visualization system. All-important data are displayed online.

- Communication with PC monitoring station - the control system sends data and receives commands from $\mathrm{PC}$ monitoring station using wireless communication system.

The race car embedded control system uses the microcontrollers Freescale HCS12. The control system has distributed architecture that is divided into five main hardware parts:

- Race car pilot cockpit with pilot control panel - steering wheel, optimal track guidance control display and voice communication,

- Control block of the electricity generator with hydrogen fuel cell,

- Control block of electricity accumulator units with super capacitors,

- Control block of the power DC drive system,

- Interference block of physical environment conditions. 
All parts of control system are connected via CAN communication network. The wireless communication between the car and PC monitoring station is realized by data link used mixed GPRS voice and data communication technologies.

The car control system as well as the PC monitoring station are equipped by GSM terminal and differential GPS station. The PC monitoring station provides a process visualization application that is realized by SCADA system Promotic. The process visualization displays all parameters measured during the car operation, all system states, alarms, make possible to display trends of required values, and log measured data in data archives.

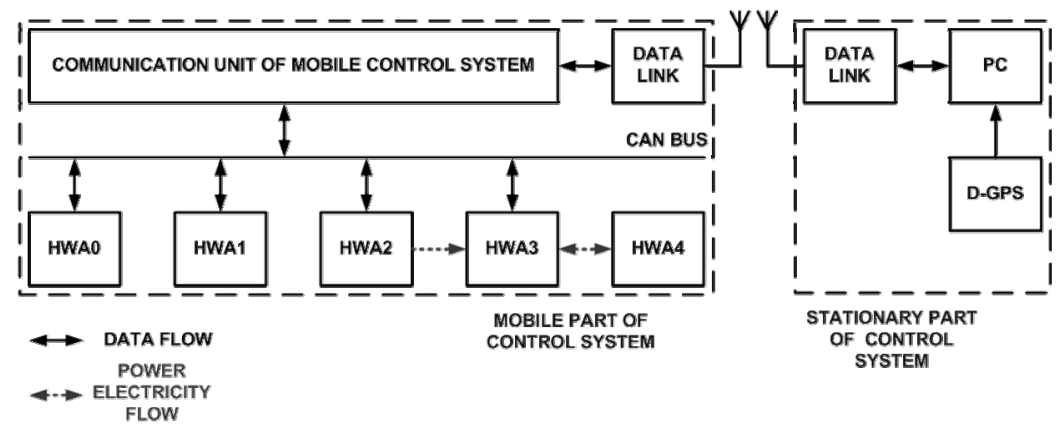

Fig. 2. The block scheme of the race car stationary and mobile part of control system, HWA0 pilot, cockpit control unit, HWA1 - physical condition description unit, HWA2 - generator of electric power, HWA3 - energy accumulator unit, HWA4 - unit of power drive, recuperation

The complete block diagram of the car control system is demonstrated in figure 2 and a laboratory realization in figure 3 .
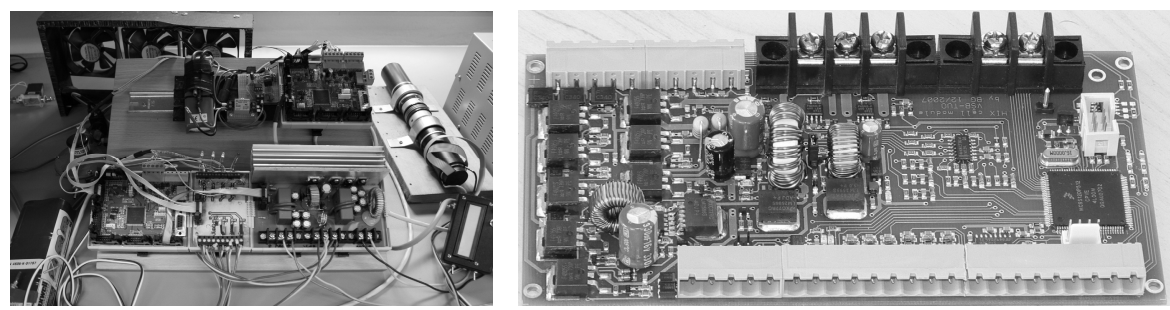

Fig. 3. The HydrogenIX car control electronic testing workplace (on the left) and final realization of control unit

\subsection{Operating Values Monitoring}

The car control system monitors many variables [3]. Some of these variables are used for basic control activities, the others are used for optimization of operation. The measured variables are following:

- Electrical variables - fuel cell voltage and current, super capacitors voltage and current, DC drive voltage and current and on-board battery voltage and current. 
- Non-electrical variables - temperatures (exterior air, cockpit, cooling air, fuel, and fuel canister), pressures (air, fuel), car speed, wind speed and wind direction, air humidity, race track position.

In figure 5, are shown graphs of chosen quantities on time archived by control system during testing runs. Testing run 1 (Fig. 4a) shows the case of periodic changing of the velocity by driver. Testing run 2 (Fig. 4b) shows a start with maximum power and then velocity set to constant value.
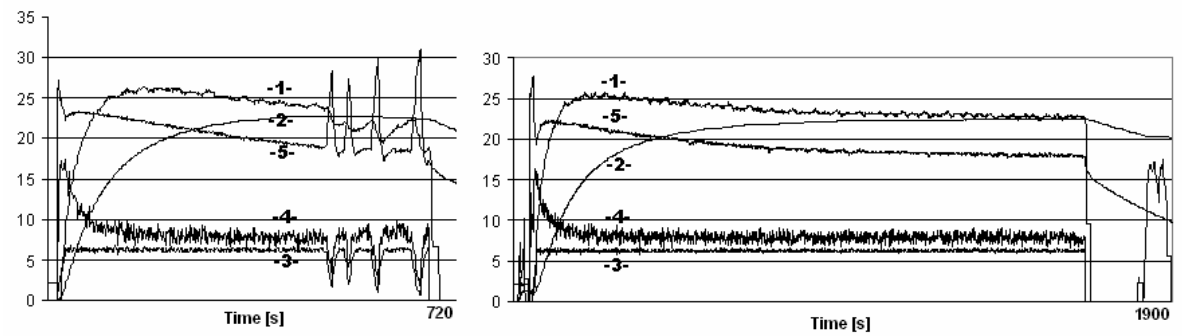

Fig. 4. a) Testing run 1 . Instant velocity $[\mathrm{km} / \mathrm{h}](1)$, average velocity $[\mathrm{km} / \mathrm{h}](2)$, Fuel cell current [A] (3), Motor current [A] (4), Fuel cell voltage [V] (5) b) Testing run 2. Instant velocity $[\mathrm{km} / \mathrm{h}](1)$, Average velocity $[\mathrm{km} / \mathrm{h}](2)$, Fuel cell current [A] (3), Motor current [A] (4), Fuel cell voltage [V] (5).

The vehicle is also equipped by bio-telemetry system that makes possible to monitor biological functions of the pilot. For biophysical monitoring was chosen pulse frequency, body temperature, respiration frequency, electrocardiography - ECG.

\section{Concept of Multi-agent Control System}

Optimization task of the racetrack passage is quite complex, the laboratory car has to consummate a minimal energy controlled in real time. Range of inputs and outputs of the control system, communication flows and safety of all operations require good level of adaptability with environment changes and event situations - base of the strategic control.

The strategic control includes number of components. The related subject should know what result is preferred. It should be able to react at intentions and actions of other participants of the situation. It should be capable of cooperation and compromise searching where it is rational [1] and [2].

Basic subject of strategic control is called an agent. The agent is software and/or hardware entity created with the aim of autonomous solving of tasks with respect at environment in which they occur. His functionality - internal action depends at communication with neighbourhoods (e.g. with sensors, actuators or other agents). Functionality is therefore given by its tasks, targets, behaviour and states. An intelligence of the agent represents often the capability of conformity, development of interactions with neighbourhoods, quick learning, data accumulation and self analysis. Multiagent systems (MAS) increase flexibility, efficiency and provide learning capability 
of new reactions and behaviour. In comparison with classic technologies the learning is a „new feature“ [4]. In learning process is possible distinguish methods on-line and off-line learning. During off-line learning process is possible to set-up of parameters and databases of system before ride. During on-line learning process learn the agents during ride. Agents can modify own behaviour by method test/mistake. Problem is in acquisition of right training set, that cover all possible situations.

Among expected basic properties of proposed MAS belong a strategic, targeted system behaviour, robustness and adaptability at environment changes. This can be provided by decentralization of control activities in the control system, by distribution of functions and by modularity based on fundamental elements - agents [1].

The agent system has a common target - safe realization of seven rounds through racing circuit in total time near of 50 minutes with minimal fuel consumption. For successful assertion of the race strategy the extraction and knowledge of changeable environment and learning capabilities are very important.

Planning of actions for accomplishment of common target is based on information about track - racing circuit and about internal state of individual agents. The influence at the fuel consumption have track geometry (length, inclination / declination, cross fall), weather (temperature and air-pressure, force and wind-direction, air-humidity, waterless / damp / soaked track), car (aerodynamic resistance, rolling and frictionresistance).

It is possible to characterize targets of individual hardware agents:

- HWA0 - pilot (maintaining of the drive direction, passive car brake, start/stop of the race car).

- HWA1 - physical condition description unit (measure the physical quantities of environment).

- HWA2 - generator of electric power (fuel-saving generation of electricity).

- HWA3 - energy accumulator (optimal select of energy source for consumption, optimal refill of sources with electric power).

- HWA4 - unit of power drive and energy recuperation (optimal control of electricity consumption).

\section{MAS Structure Description}

The MAS structure block scheme is shown at figure 5. The higher level of control system is represented by a personal computer. Differential GPS positioning system represents the relative coordinate system of environment - allow to precise of the position of the race car on the circuit. The GPRS communication modem is connected at the output - data link, which transmits commands for race car embedded mobile control system.

The separate task is the transformation which converts the global digital data (inclination, declination, wind speed and wind direction) and digital data of the position into the object coordinates (car position on the circuit), which are saved in the database of the circuit. This database is common for all agents in the control system. 


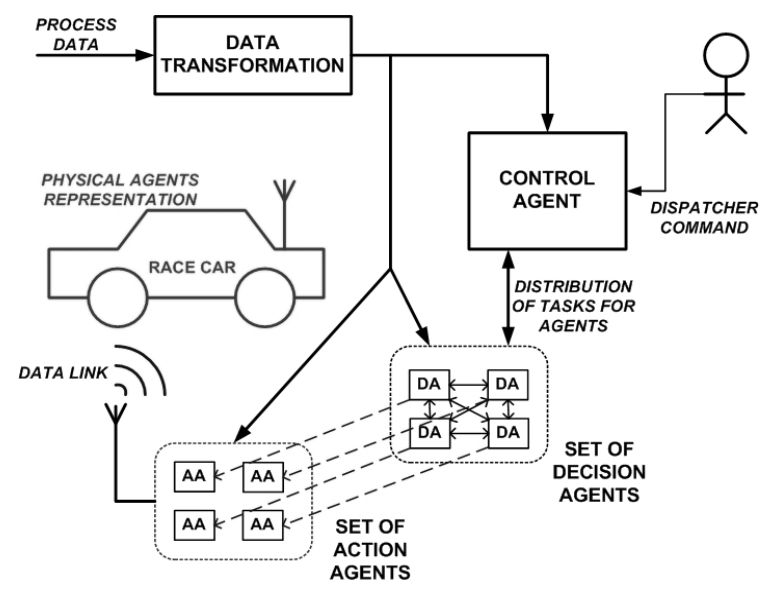

Fig. 5. Block scheme of Multi-Agent control system concept

Each agent access to the all actual data and is capable to control its behaviour in the qualified way. The basic characteristic of a control algorithm of a subordinate agent is independence on the number of decision making agents for car on the circuit.

Main architecture of such hybrid agent system is characterized by:

- Layered control. An agent is described by number layers of abstraction and complexity.

- Layered knowledge base.

- Bottom-up activating.

- Top-down execution.

The algorithm of agent's cooperation was proposed by the control agent on a higher level. The control agent (Fig. 6) goals (minimize fuel consumption) can be achieved by the correct selection of cooperating agents (division of tasks among agents). The decision making agents are able to select correct tasks for themselves and further to select executive agents.

The sensation module evaluates the actual state of the data. The data does not describe the position of car but gives the information about the capability of car to move with optimal trajectory and optimal velocity. In the case that the dynamic control and decision making system will not find the solution for a certain situation, then it will try to find the partial solution from several situations. The controller selects the relevant tasks for the agents for these situations. The controller saves or updates the information about the scene state and control in the relevant database which can be used for static control.

The main task of the decision agent (Fig. 7) is to schedule the relevant task. The agent will schedule the tasks in relation to the environment and the internal state of the relevant hardware unit. The module of perception provides the information about the environment. 


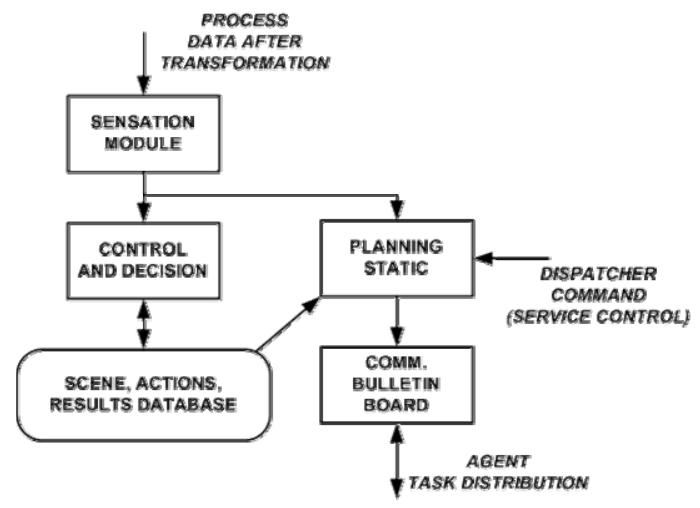

Fig. 6. Control agent scheme

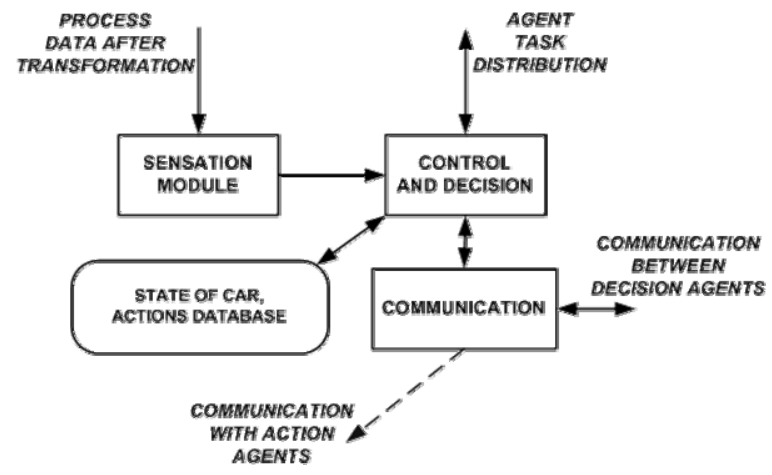

Fig. 7. Decision agent scheme

In the case that more than one agent will select the same task the decision agent must evaluate the probability of the action's success. The agent with the highest probability of success will schedule the actual task. The task selected is handed over to the executive agent who realizes it. The executive agent will receive also the information from cooperating agents for optimization of the car movement.

The activity of the action agent (Fig. 8) is simple. The agent moves with the race car from an actual position to a new position. The information concerning the actual position is obtained from the sensation module.

A new position for further moment (for instance another frame of the GPS data) is evaluated by the control module. The new position is calculated on the basis of a precalculated trajectory. After completion of the trajectory calculation the agent will determine the position for next frame (new position) and will transmit it to the action module.

Agents are connected with the environment through interface realized by sensors, actuators and communication module units (HWA0-4, D-GPS). Control is divided to the layers they use information from knowledge bases [2]. 


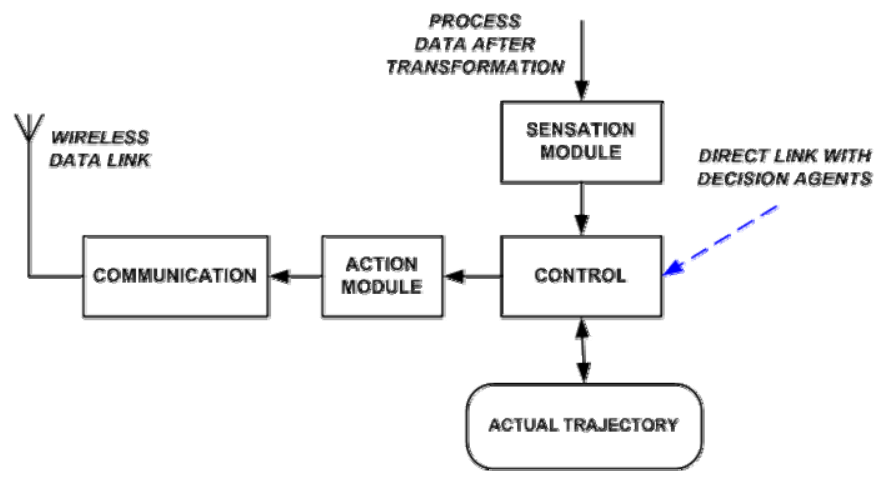

Fig. 8. Action agent scheme

Local planning layer. Some situations cannot be solved by execution of template action like an answer to stimulation from environment only, but they require certain deliberation level. A function of plans creation for solving of the targets performs the layer of local planning. Local planning layer has such fundamental data structures:

- Targets - are defined by state sets. The sets are characterized by attributes that are fulfilled at reaching targets.

- Planning - principles of planning. Sets of plans are predefined as data structures plans library. Target sets are mapped into the plans library. For each target is possible to assign the plan for its reaching.

- Plans library - contains the plans for reaching of agent targets.

- Scheduling - secures time-limited plans stratification. Created plan schedules are executed like the step sequences.

Cooperative planning layer. A basic control cycle of cooperative planning layer is creation, interpretation, decision making and execution of local plans. In first phase the reports from nearby layers are processed. Reactive layer sends requests to solve new task or status of executed behaviour templates. Schedules of active plans are actualized. Subsequently the status of reactive layer executed procedures is checked.

In case of successful procedures finalization the plan is erased from accumulator. Reports from highest layer are related to creation or cancellation of commitment for the plan execution at local base or plan evaluation. In case of plan execution request or its cancellation the accumulator of active plans is actualized.

The plan availability is a result of difference between its relative value for the agent and its execution costs. The plan value is derived from target value that is possible to reach by plan. The plan costs are determined by function that assigns to every plan a real number calculated at basis of its fundamental action costs according to specific rules.

In gradual hierarchy of targets, it is not possible to realize the target without coordination with other agents. The purpose of multi-agent planning is in "unlocking" such target through building of common plan. Unlocking the target doesn't explicit it's reaching. It's sufficient if agent can continue after execution of common plan by tracking of own local plans. Common plans contain actions of all agents without 
synchronizations that are not directly executable. It is important to transform common plans into executable plans for individual agents.

Reactive layer. It is responsible for adequate reactions to the stimulations from environment that requires the immediate reaction and execution of called procedures from local planning layer. Fundamental characterization of such layer is:

- Use of effective algorithm of comparison with patterns of behaviour. Serve to pickout of the actual situations.

- Situation description for timely actual reactions at received stimulus.

- Hard-wired links. Recognized situations are fix-connected with targets for reactive behaviour. Immediate execution of program actions.

- Solution request of situations not-corresponding with couple's situation-action is transmitted in local planning layer.

- Execution liability is coming from local planning layer to activate procedures of reactive layer patterns of behaviour.

\section{Conclusions}

The algorithm of the control system should be proposed in a way to ensure the requirements for the immediate response of control so that the system of race car would be controlled in real-time. That is why, it is very important that the algorithm for the critical highest speed, the critical average speed, the dead-line time and the fuel (stored energies) consumption would be optimized. The system response should be shorter than the sensitivity (position error) and/or time between two data frames from a GPS station. In the case that this limit is exceeded, the control quality may be decreased.

The main features of algorithm adjustment are following:

-Dynamic control in the control and decision module of a control agent.

-The control and decision modules and communication protocol of the decision agents.

-The strategy of planning in the control model of the action agent.

-Learning of a race strategy and using the extraction results for decision rules generation as a part of the rules decision database of a decision agent.

Acknowledgments. The Grant Agency of Czech Academy of Science supplied the results of the project No. p. 1ET101940418 with subvention.

\section{References}

1. Pavliska, A., Srovnal, V.: Robot Disassembly Process Using Multi-agent System. In: Dunin-Keplicz, B., Nawarecki, E. (eds.) CEEMAS 2001. LNCS (LNAI), vol. 2296, pp. 227-233. Springer, Heidelberg (2002)

2. Srovnal, V., Horak, B., Bernatik, R., Snasel, V.: Strategy Extraction for Mobile Embedded Control Systems Apply the Multi-agent Technology. In: Bubak, M., van Albada, G.D., Sloot, P.M.A., Dongarra, J. (eds.) ICCS 2004. LNCS, vol. 3038, pp. 631-637. Springer, Heidelberg (2004) 
3. Garani, G., Adam, G.: Qualitative Modelling of Manufacturing Machinery. In: Proceedings of the 32nd IEEE Conference on Industrial Electronics, pp. 3591-3596. IEEE, Paris (2006)

4. Kaelbling, L.P.: Learning in Embedded Systems. MIT Press, Cambridge, Mass (1993)

5. Vilaplana, M.A., Mason, O., Leith, D.J., Leithead, W.E.: Control of Yaw Rate and Sideslip in 4-wheel Steering Cars with Actuator Constraints. In: Murray-Smith, R., Shorten, R. (eds.) Switching and Learning 2004. LNCS, vol. 3355, pp. 201-222. Springer, Heidelberg (2005)

6. Turek, W., Marcjan, R., Cetnarowicz, K.: Agent-Based Mobile Robots Navigation Framework. In: Alexandrov, V.N., van Albada, G.D., Sloot, P.M.A., Dongarra, J. (eds.) ICCS 2006. LNCS, vol. 3993, pp. 775-782. Springer, Heidelberg (2006) 